\author{
ANNALS OF “DUNAREA DE JOS” UNIVERSITY OF GALATI \\ MATHEMATICS, PHYSICS, THEORETICAL MECHANICS \\ FASCICLE II, YEAR X (XLI) 2018, No. 2
}

Article DOI: https://doi.org/10.35219/ann-ugal-math-phys-mec.2018.2.01

\title{
MULTI- AND INTER- DISCIPLINARY APPROACHES IN THE SCIENTIFIC DOCUMENTATION AND MONITORING OF CULTURAL HERITAGE ASSETS
}

\author{
Laurențiu-Marian Angheluță ${ }^{1 *}$, Roxana Rădvan ${ }^{1}$ \\ I*National Institute of Research and Development for Optoelectronics INOE 2000; laurentiu@inoe.ro
}

\begin{abstract}
This paper synthesizes several aspects of the trends of the multi- and inter-disciplinary strategies regarding both research and applications in the scientific documentation and conservation status monitoring of cultural heritage assets such as museum items, monuments, archaeological sites or even larger cultural landscapes, using modern technology. A comprehensive understanding of all physico-chemical, natural, social, and historical factors that had or still have damaging effects on the cultural heritage assets is necessary and requires a different approach to the hyperspecialization mindset that tends to take over the academic disciplines. All these factors are interrelated and cannot be separated nor can a preventive conservation strategy be successfully implemented by considering just one aspect of the problem. Along this line this paper presents our own solutions and their evolution during time. Multi-disciplinary projects in collaboration with research groups coming from different disciplines (natural studies or social and humanities areas) will be discussed. E-learning courses for joint groups of students coming from different disciplines will also be described together with online platforms with customized databases for the management and dissemination of the scientific data resulted from the project activities.
\end{abstract}

Keywords: cultural heritage, scientific collaboration, online platforms, conservation strategy, integrated research

\section{INTRODUCTION}

Terms like multi-disciplinary, inter-disciplinary, cross-disciplinary or trans-disciplinary are increasingly used in research reports, funding calls or scientific literature, all regarding different levels of integrated research. We are witnessing an integration stage of technology evolution where everyone and everything is inter-connected and inter-related. All these changes came in a short period of time and, although the research community is aware and already aligning themselves with this new approach, there are many definitions for the afore-mentioned terms leading to obvious misunderstandings.

Zeigler outlined back in 1990 [1] and A.R. Jensenius in 2012 [2] the differences between collaborative modalities in medical research in a diagram that we have updated to a more current context (see Fig. 1). 


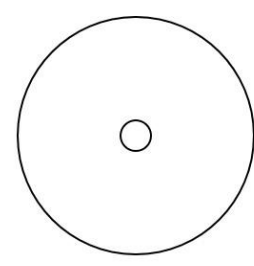

Intra-disciplinary

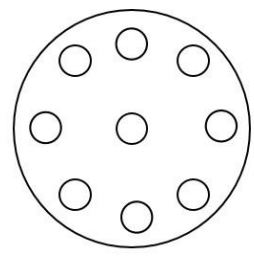

Multi-disciplinary

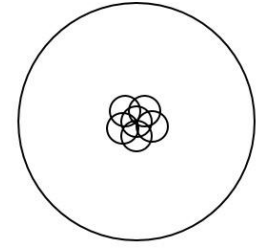

Inter-disciplinary

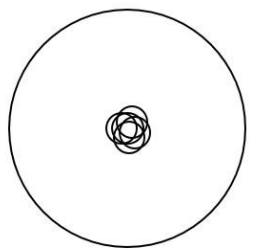

Trans-disciplinary

Fig. 1: Understanding the different types of relationships between disciplines in an integrated research project

One of the general misconceptions regards inter-disciplinarity and multi-disciplinarity. Multidisciplinarity is characterized as a collaboration of several academic disciplines in a specific themed investigation with multiple purposes. Within such collaborations the researchers are sharing their own results and knowledge without crossing the "boundaries" in order to generate new knowledge or to solve a common problem. There are debates whether in this type of approach the research is coordinated or integrative at low level [3]. Inter-disciplinarity, on the other hand, requires a much more collaborative approach to a problem. For example a conservation-restoration modern laboratory with state of the art equipment requires an inter-disciplinary team in order to address the complex casuistry of different types of materials and objects that they are monitoring/investigating. In some cases specialized inter-disciplinary laboratories carries out multi-disciplinary projects in collaboration with museums, art galleries or other cultural heritage administrators.

Perhaps the most difficult form of integrated research to obtain is trans-disciplinarity. A simple definition of trans-disciplinarity would be "a process of collaboration between scientists and non-scientists on a specific real-world problem" [4]. The goal of such endeavour typically is to generate a decision-making policy (or capacity) of the involved stakeholders. This kind of projects cannot be realized without the involvement of non-academic partners like land managers, public institutions, user groups or the general public [3].

This paper describes several examples of these forms of integrated research applied in the documentation and monitoring of cultural heritage assets, with examples from our own past projects and experience.

\section{CONTEXT AND METHODS}

Cultural heritage assets are in permanent exposure to damaging risks and usually need a complex strategy for the preventive conservation policy. Whether the subjects are part of a cultural landscape in open air, protected by improvised constructions, open to the public or stored in a secure and controlled environment, their maintenance and documentation requires complex strategies. A complete documentation regarding the conservation status of such a subject involves an interdisciplinary approach and should include a large variety of state of the art investigation and documenting methods coming from different disciplines.

As it was mentioned before, a scientific laboratory than can tackle most of the critical issues regarding the investigation and high precision documentation of artworks' conservation state must be inter-disciplinary. The required knowledge and know-how for this type of activity comes from different branches of the natural sciences: physics, chemistry, biology, geology, history. Usually natural sciences work hand in hand with applied sciences disciplines like engineering or education in order to generate powerful tools and devices for documentation, investigation and intervention. Each of these disciplines specializations alone cannot be self-sufficient in determining a full and correct characterization of the material quality, body structure, pigment composition, etc. They must be corroborated so that each aspect of the conservation problem can be covered and fully documented. 


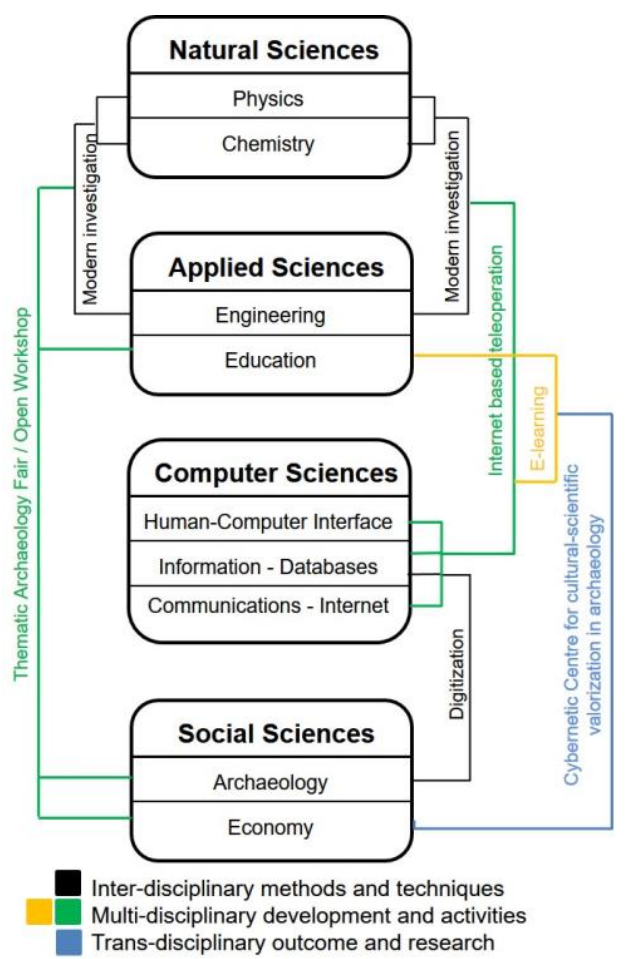

Fig. 2. The relationship between the involved disciplines in a transdisciplinary project (2013-2017)

Having the technological aspect covered allows us to correctly asses the conservation state of a cultural heritage asset, but is it enough? Figure 2 depicts a schematic of the inter-connectivity levels between different disciplines during a trans-disciplinary project elaborated and developed by a multidisciplinary consortium consisted in two history museums and a research and development institute for optoelectronics (with a specialized department for modern conservation methods in cultural heritage) [5]. This is an example of how far multi-disciplinarity can go in order to create products that transcends the scope and limitations of each of the involved party: joint workshops, e-learning courses for students or the know-how transfer for the design of a local cybernetic center for cultural-scientific valorization in archaeology. These activities involve both the specialized partners in the project and the local institutions, general public and certain user groups (students from different disciplines).

In the last decade, the Computer Sciences discipline played more and more a crucial role in the development of critical tools for processing, management, dissemination and promoting the scientific data. This new branch to intertwine with offered new and faster options and solutions for (not only) cultural heritage digital data. Modern approaches like online digital platforms allow the scientific teams to manage, store and display never-before online accessible data such as pigment and material characteristic data-sets, interactive high precision 3D models or multi-layer aerial survey maps. But this concept did not appear overnight. Projects like CULTURE 2000: Advanced on-site laboratory for European Antique Heritage Restoration 2005-2006 [6] led to ART4ART - Advanced Research Technology for Art and Archaeology 2008 [7]. This particular project helped redefining the concept of on-site investigation with an active modular mobile laboratory, that allows a synchronized investigation with multiple complementary technologies (3D laser scanning, laser induced fluorescence scanning, digital microscopy, laser Doppler vibrometry, microclimate monitoring, thermal and multispectral imaging etc.). This concept was further developed and improved in such projects as WWWwATCH: World Wide Open Workshop with Advanced Techniques for Cultural Heritage [8], started in 2012, which is taking the concept of integrated multidisciplinary investigations to a whole other level, by developing for the first time in Romania an online platform for both real time access to the mobile infrastructure (teleoperation) and field campaign data management in an online database. While consolidating this kind of infrastructure and gathering large sets of 3D laser 
scanning investigations (Basarabi-Murfatlar cave complex, Buddhist temples from Sasspol and Ladakh - Tibet, Bucovina monasteries, etc.), it was mandatory to develop a multilayered data model generated by inter-disciplinary research efforts, a model that can be adapted also to other type of cultural heritage categories [9].

Regarding the integration of 3D models for online interactive visualization, several steps were made in this direction, starting with a low budget project Arheodrom Virtual 2010 [10,11], where four Dobrudjan painted tombs $\left(4^{\text {th }}-6^{\text {th }}\right.$ century A.D.) were investigated with two non-invasive complementary techniques (thermal imaging and laser Doppler Vibrometry), overlaid on their respective 3D digital models, obtained by means of 3D laser scanning. It was the first time when an interactive digital model of an historical monument was available online along with overlaid imagistic results [9]. This type of visualization is currently used, but on a larger scale, at the Ulpia Traiana Sarmizegetusa archaeological site, within CERES: Center for the valorization of cultural-scientific projects in archaeology" [5, 12] and the Rupestral cave complex from Buzău Mountains in "ARTEMIS: Platformă digitală integrată pentru valorizarea peisajului cultural din Munții Buzăului. Studiu de caz: cadrul natural și monumentele istorice din zona Aluniș-Bozioru" [13] projects. Both these projects delivered two online platforms (in 2017 and 2018) with personalized databases to manage and disseminate the research data coming from multi-disciplinary teams (archaeological, biological, soil and geology, environmental monitoring, conservation state documentation) along with the interactive 3D digital models support. The latest in this category line of projects is currently under development and is the most complex so far. The Project Implement [14] involves no less than six institutions in the field of artwork conservation and is structured in five smaller projects coordinated by five of the six partners. The aim of this project is to improve the research capacity of each partner while emphasizing the trans-disciplinary research capacity, specialized infrastructure and the performance implemented through the projects in the last years. One of Implement's central goal is the development of a new online digital platform that is able to serve a broad range of specialists from any field related to the conservation of cultural heritage goods. Similarly to the previous instruments, this tool is also based on a database but this time the structure of the managed data is based on an exhaustive universal data sheet that can be corroborated with any form of international database in this field.

\section{RESULTS}

This paper does not go into details about the means of networking and creating partner clusters, much needed for this type of multi-disciplinary collaborations, because each research entity is based on such protocols and it would be a different kind of discussion. Instead the focus is set on the deliverables and the means to obtain them. As one can observe from the previous section, this kind of projects have some deliverables in common: open-site workshops, courses and e-learning sessions or online platforms.

Open-site workshops are usually coupled with basic courses for students or specialized personnel that want to expand their knowledge above their expertise. Such events are organized during a field data acquisition campaigns, on site. In a multi-disciplinary collaboration it is a common process for the involved teams to organize this kind of workshops for a better know-how exchange while working in-situ. For an extended access these sessions can be transmitted live through video over the Internet. E-learning events are usually performed in-situ or in-lab for classes of students or groups of researchers from abroad. These sessions are usually recorded so they can be used for offline learning. The topics that these e-learning sessions and live courses are taught with a multi and interdisciplinary approach in mind in order to further emphasize the importance and normality of data and methods corroboration.

Online platforms are databases customized for the casuistry of each project. These databases are used for the management, storage and ease of access for investigation results and documentation data obtained for a set of artworks. Usually, these platforms are designed to cover the data typology of multi-disciplinary investigations. To the ease of use for both project operators and the targeted public, these databases are accessed through a user-friendly graphic interface in a web browser. These user- 
interfaces are always connected to a project presentation website and, in most of the cases the information within these databases is accessed by authorized users and can be update only by designated operators.

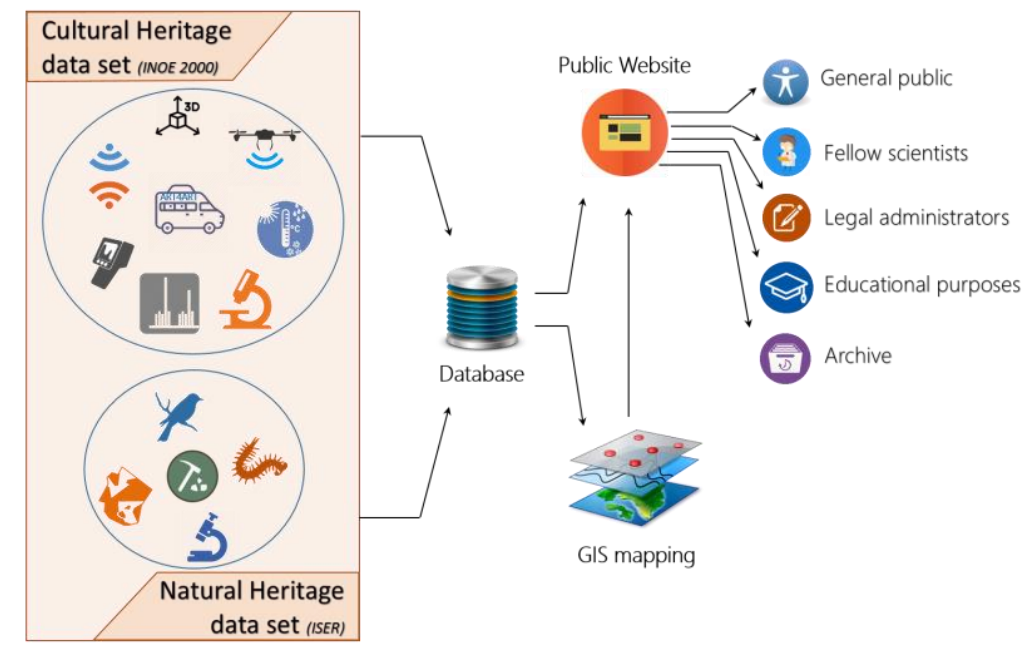

Fig. 3 Online platform schematic for the ARTEMIS project [15]

Figure 3 depicts such an example that follows the flow of information from data acquisition to the target public in the ARTEMIS project. In this project the multi-disciplinary team was consisted of two groups with different fields of research: the natural heritage on the one hand and the cultural heritage on the other. The developed platform is able to manage and display data sets coming from these two different fields: birds, amphibia and invertebrates monitoring, environment micro-climate monitoring, geology studies for the rock quality in the rupestral caves areas, 3D digitization of the monitored monuments, technical and multispectral photography and aerial survey with several types of imaging sensors. All these different types of data are integrated in a way that is easy for any following researcher to corroborate and compare future data. In addition, a separate section is dedicated to an interactive multi-layered map of the studied area. The map layers are recomposed mosaics of aerial survey data coming from thermal, photogrammetry, lidar and multispectral imaging sensors.

Another example is the online platform for the PILOT project. Figure 4 shows a typical interconnectivity between the consortium partners for the development of an online instrument for the management of archaeological deposits. This platform is generated as a product based on the associated knowledge from physics, chemistry, optical engineering, museology, conservationrestoration, but is also extrapolated to other specific applications for archaeology, visual art, archive etc. A synergy of the consortium is ensured by each partner's contribution with data and experience in complementary fields, that serves for the complete description of a deposit with high cultural value, containing both physico-chemical and historical descriptions.

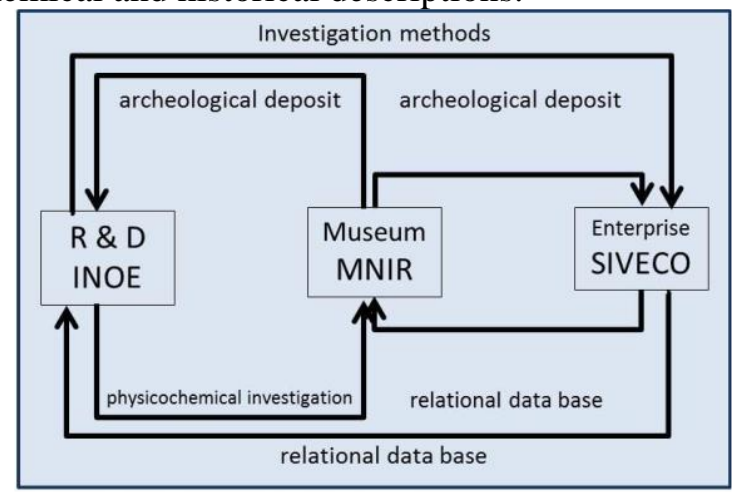




\section{Fig. 4 Inter-connectivity relationships between project partners for the development of a dedicated online platform for archaeological finds}

As for the development tools, there are no restrictions or limitations. The presented projects developed their platforms mostly by basic web development frameworks like PHP, HTML and MySQL, but friendlier frameworks like WordPress or Joomla can also be successfully used.

\section{CONCLUSIONS}

Interpolating the descriptions and the results of the presented multi- and trans-disciplinary projects in the field of Cultural Heritage, we can summarize a few aspects regarding the integrated research forms:

- Inter-disciplinarity refers to a deep connection between inter-related disciplines in order to solve a problem and to create a new means of research for that specific problem;

- Multi-disciplinarity can be described as a collaboration between specialized or interdisciplinary groups in order to sum up different knowledge about a common set of problems, each group thus contributing with its own area of expertise;

- Trans-disciplinarity can be thought as the ultimate goal of the integrated research forms. It involves all the aforementioned forms of research by crossing the disciplines boundaries in order to tackle and solve real-world problems by implementing real-world solutions with the help of nonacademic third party.

The integrated research models based on inter-, multi- and trans- disciplinarity determine new coherent approaches on the valorisation process of the cultural heritage assets by corroborating complex scientific data and know-how (physics, chemistry, biology, geology) from different disciplines with complementary knowledge from humanities and social sciences (archaeology, anthropology, sociology, etc.). Involving direct and indirect beneficiaries in the project development process transposes the coordinates of scientific activities and results into the real world solving real, tangible problems.

\section{Acknowledgement}

This work was supported by a grant of the Romanian Ministry of Research and Innovation, CCCDI - UEFISCDI, project number PN-III-P1-1.2-PCCDI-2017-0878/NR. 55PCCDI/2018, within PNCDI III. References

1. Zeigler, E.F. Professional Preparation and Discipline Specialization in Canadian PE and Kinesiology. Journal of Physical Education, Recreation \& Dance, 1990, 61, 40-44.

2. Blog - Alexander Refsum Jensenius. Available online: http://www.arj.no/2012/03/12/disciplinarities-2/ (accessed on 09.10.2018)

3. Paul S., Rob J.F.B. Defining Terms for Integrated (Multi-Inter-Trans-Disciplinary) Sustainability Research, Sustainability, 2011, 3, 1090-1113.

4. Walter, A.I.; Helgenberger, S.; Wiek, A.; Scholz, R.W. Measuring societal effects of transdisciplinary research projects: Design and application of an evaluation method. Eval. Program Plann., 2007, 30, 325-338.

5. Project CERES. Available online: http://certo.inoe.ro/ceres/ro/index.php (accessed on 09.10.2018).

6. Project Culture 2000. Available online: http://constanta.inoe.ro/ (accessed on 09.10.2018)

7. Project ART4ART. Available online: http://art4art.inoe.ro/index.html (accessed on 09.10.2018).

8. Project WWWwATCH. Available online: http://certo.inoe.ro/watch (accessed on 09.10.2018). 
9. Angheluță L.-M., Ene D. V., Moldovan A.-S., Modern Method for the Visualization of Photonic Investigations. Case study: Painted Tombs in Constanta, 44th edition of Pontica Conference, 2011 Constanța

10. Arheodrom virtual. Available online: http://www.minac.ro/arheodrom/ (accessed on 09.10.2018).

11. Certo virtual. Available online: http://certo.inoe.ro/virtual (accessed on 09.10.2018)

12. Angheluță, L. M., Rădvan, R., Chelmuș, A. I., Ratoiu, L., Cortea, I. M., and Ghervase, L.: Virtual Archaeodrome for the Archaeological Site from Ulpia Traiana Sarmizegetusa, Int. Arch. Photogramm. Remote Sens. Spatial Inf. Sci., 2017, XLII-5/W1, 351-356, https://doi.org/10.5194/isprs-archives-XLII-5-W1-351-2017

13. Project ARTEMIS. Available online: http://certo.inoe.ro/artemis/ (accessed on 09.10.2018)

14. Project IMPLEMENT. Available online: https://implement.inoe.ro/ (accessed on 09.10.2018)

15. Angheluță, L. M., Ratoiu, L., Chelmus, A. I., Rădvan, R., and Petculescu, A.: Integrated Digital Platform for the Valorization of a Cultural Landscape, Int. Arch. Photogramm. Remote Sens. Spatial Inf. Sci., 2017, XLII-5/W1, 389-393, DOI: 10.5194/isprs-archivesXLII-5-W1-389-2017 